BNL- 45959

\title{
Populating 114 Buckets by Bunch Shifting Study on Cottingham's Scheme
}

\author{
J. Wei and A. G. Ruggiero
}

March 1991

\section{DISCLAIMER}

This report was prepared as an account of work sponsored by an agency of the United States Government. Neither the United States Government nor any agency thereof, nor any of their employees, makes any warranty, express or implied, or assumes any legal liability or responsibility for the accuracy, completeness, or usefulness of any information, apparatus, product, or process disclosed, or represents that its use would not infringe privately owned rights. Reference herein to any specific commercial product, process, or service by trade name, trademark, manufacturer, or otherwise does not necessarily constitute or imply its endorsement, recommendation, or favoring by the United States Government or any agency thereof. The views and opinions of authors expressed herein do not necessarily state or reflect those of the United States Government or any agency thereof.

\section{RHICPRO J E C T}

\author{
Brookhaven National Laboratory \\ Associated Universities, Inc. \\ Upton, NY 11973
}




\title{
Populating 114 Buckets by Bunch Shifting \\ - Study on Cottingham's Scheme
}

\author{
J. Wei and A.G. Ruggiero \\ Accelerator Development Department \\ Brookhaven National Laboratory \\ Upton, New York 11973
}

\begin{abstract}
Cottingham proposed a scheme to populate 114, instead of 57 , rf buckets at the RHIC injection, which employes a low- $Q$ rf system of maximum $40 \mathrm{kV}$ in addition to the original rf system to shift the particle bunclies azimulhally along the ring.

The first part of this report studies the adiabatic condition required by this scleme. It is shown that the entire process can be accomplished during a time shorter than the cycling period of the AGS. Results of computer simulation, which are presented in the second part, indicate that particle loss is negligible during the manipulation of the $0.3 \mathrm{eV} \cdot \mathrm{s} / \mathrm{u}^{197} \mathrm{\Lambda u} \mathrm{u}^{79+}$ bunches.
\end{abstract}




\section{Introduction}

According to the Conception Design Manual, 57 of the 342 buckets produced by the If accelerating system in the RHIC are to be populated ${ }^{1}$ by the particle bunches injected from the AGS. Recently, several schemes have been proposed to increase this number from 57 to 114 .

One of the schemes, which is proposed by Cottinghan ${ }^{2-4}$, employes a low- $Q r[$ system of maximum $40 \mathrm{kV}$ in addition to the original $h=342$ high- $Q$ system. Every time after 3 bunches are injected into the RHIC, this low-Q system is switched on which, combined with the original system, provides moving buckets that shift the injected bunches azimuthally along the ring. Using this method, the distance between successive bunches can be reduced from 6 to 3 bucket widths.

This report studies the theoretical aspects of this scheme. The adialuatic condition required for the bunch shifting is derived in section II. Results of computer simulation is presented in section III.

\section{Condition of Adiabaticity}

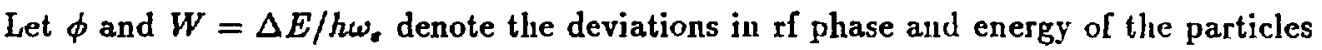
from the synchronous values relative to the high- $Q$ if system, where $\omega_{a}$ is the synchronous revolution frequency, $E$ is the total energy of the particle, and $h$ is the rf harmouic number. During each revolution, the voltage of the high- $Q$ system expericnced by the particle is

$$
V_{1}=\hat{V} \sin \phi
$$

where $\hat{V}$ is the peak voltage.

The low- $Q$ voltage $V_{2}$ applies only on the bunches that is required to be shifted. The proposed voltage to achieve azimuthally sinifting of buckets is

$$
V_{2}=-2 \dot{V} \sin \frac{\phi_{d}}{2} \sin \left(\phi+\frac{\pi}{2}-\frac{\phi_{d}}{2}\right)
$$


where $\phi_{\alpha}$ is linear in time $t$,

$$
\phi_{d}=-v_{d} t
$$

with $v_{d}$ a constant. The timing of this low-Q rf system has to be controlled in accordance with the locations of the bunches inside the ring.

For particles of the bunches that are to be shifted, the total voltage experienced each revolution is

$$
V=V_{1}+V_{2}=\hat{V} \sin \left(\phi-\phi_{d}\right)
$$

This voltage provides buckets that move azimuthally in $\phi$ with a speed $v_{d}$. The longitudinal motion of the particles under this voltage can be described by

$$
\left\{\begin{array}{l}
\dot{W}=C_{\phi} \sin \left(\phi-\phi_{d}\right) \\
\dot{\phi}=C_{W} W
\end{array}\right.
$$

where

$$
C_{\phi}=\frac{q e \dot{V}}{2 \pi h}, \text { and } C_{W}=\frac{h^{2} \omega_{s}^{2} \eta}{E \beta^{2}}
$$

The solution to Eq. 5 is a superposition of a linear shift and that of the harmonic oscillator, which satisfies

$$
\ddot{\phi}=\Omega_{a}^{2} \sin \left(\phi+v_{d} t\right)
$$

where

$$
\Omega_{\lrcorner}=\sqrt{C_{\phi} C_{W}}
$$

is the linear synchrotron-oscillation frequency.

The crucial condition for the particles to be confined by the moving buckets and shifted azimuthally is the adiabaticity. According to the Liouville theorem, particles follow the evolution of the If buckets with an invariant phase-space area if the relative changes in the buckets are small in one synchrotron-oscillation period. If this condition is satisfied, particles near the original synchronous point

$$
\phi=\phi, \text { and } W=0 \text {, at } t=0 \text {, }
$$


shift with approximately the speed $v_{d}$ in $\phi$. The solution to Eq. 7 is then oblaincd as

$$
\phi=-v_{d} t+\hat{\phi} \sin \left(\Omega_{s} t+\phi^{0}\right)
$$

where $\hat{\phi}$ and $\phi^{0}$ are consiants determined by the initial conditions.

For instance, the motion of the original synchronous particle satisfying Eq. 9 is obtained from Eq. 10 as

$$
\phi_{d}(t)=-v_{d} t+\frac{v_{d}}{\Omega_{s}} \sin \Omega_{s} t .
$$

The second term at the right hand side of Eq. 11 indicates the deviation from a pure shift (the first term). In order to minimize this deviation, the process must be accomplished adiabatically,

$$
N_{d}=\frac{\Omega_{s}}{v_{d}} \gg 1,
$$

i.e. the number $N_{d}$ of synclirotron oscillations that corresponds to a slift of $2 \pi$ radian in $\phi$ must be much larger than 1.

\section{Results of Computer Simulation}

The program TIBETAN has been ${ }^{5}$ modified to simulate the process of azimulhal slifting of the ${ }^{197} \mathrm{Au}^{79+}$ ion bunclies. Assume that the initial total bunch area is $0.3 \mathrm{eV} \cdot \mathrm{s} / \mathrm{u}$. With the peak voltage $\hat{V}=40 \mathrm{kV}$, the pliase-space area of the slifting bucket is about $0.4 \mathrm{eV} \cdot \mathrm{s} / \mathrm{u}$. The simulation shows that to achieve a reasonable efficiency, the time to shift the bunch by $2 \pi$ radian in phase should be more than 3 times the synchrotron-oscillation period, j.c. $N_{d} \geq 3$.

Fig. 1 shows the phase-space diagram of a matched Gaussian-like bunch before the low-Q system is switched on. The low-Q system is controlled such that $N_{d}=3$, i.e.

$$
v_{d}=\frac{\Omega_{d}}{3} \approx 1.6 \times 10^{2} \mathrm{~s}^{-1} \text {. }
$$

Fig. 2 shows the correspouding diagram after 9 synchrolron-oscillation period (about $0.11 \mathrm{~s}$ ). The bunch is shifted througl a distance of 3 If bucket widths. The particle loss is negligible. 


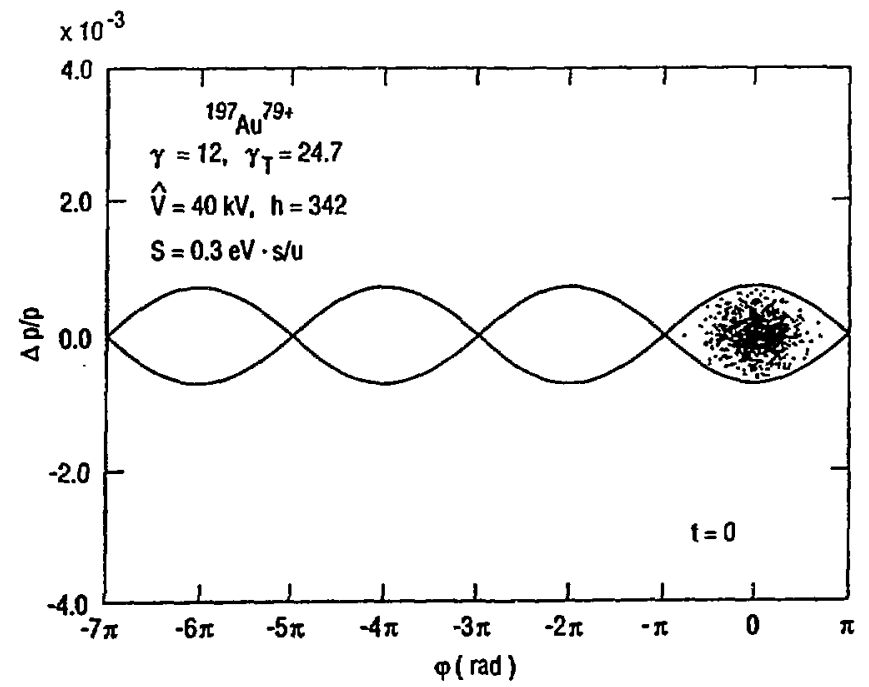

Figure 1: Longitudinal phase-space diagram of a bunch of ${ }^{197} \mathrm{Au}^{79+}$ ions with an area 0.3 $\mathrm{eV} \cdot \mathrm{s} / \mathrm{u}$, matched to the $h=342 \mathrm{rf}$ bucket of $40 \mathrm{kV}$ peak voltage.

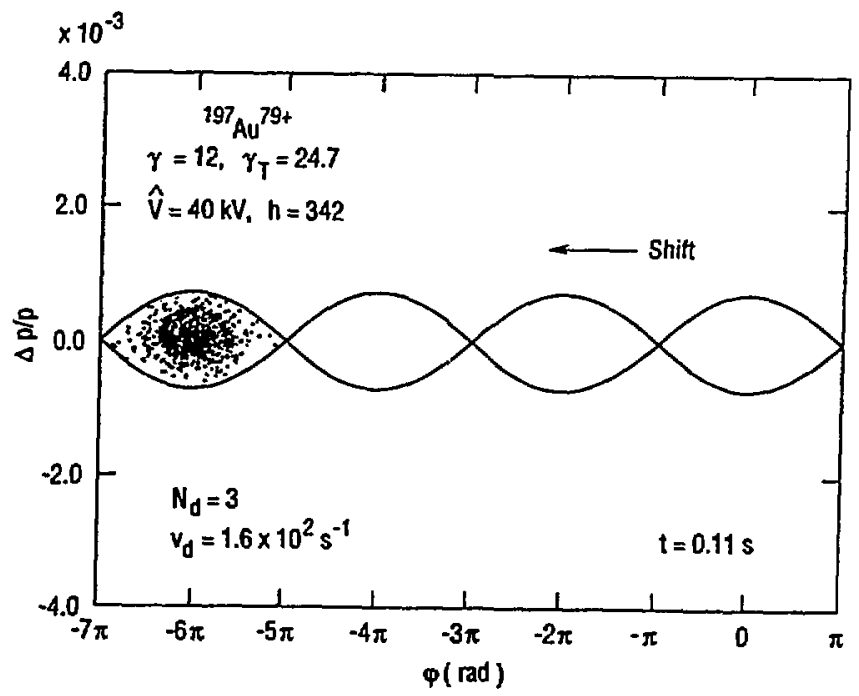

Figure 2: Longitudinal phase-space diagram of the ${ }^{197} \mathrm{Au}^{79+}$ bunch $0.11 \mathrm{~s}$ after the low- $\mathrm{Q}$ If sysiem is switched on. The bunch is shifted in phase for a distance 3 times that of the buckel width. 


\section{Discussion}

With an rf voltage of $40 \mathrm{kV}$, particles of a $0.3 \mathrm{eV} \cdot \mathrm{s} / \mathrm{u}$ bunch occupies about $3 / 4$ of the bucket area. Therefore, the buncli-shifting process should be performed sufficiently slowly to avoid particle loss. It is indicated that the process of shifting the three injecled bunclies of ${ }^{187} \mathrm{Au}^{78+}$ to the desired locations along the ring can be accomplished in about $0.5 \mathrm{~s}$, which is shorter than the cycling period of the AGS.

In order to avoid particle loss and bunch area growth, the bunches injected from the AGS should be captured by the high- $Q$ system at a peak voltage of about $220 \mathrm{kV}$ which provides buckets matched to the injected bunches. The voltage of this high-Q system is then adiabatically reduced to $40 \mathrm{kV}$ before the low- $Q$ system is switched on.

\section{Acknowledgment}

The authors would like to thank J.G. Cottingham for helpful discussion.

\section{References}

1. Conceptual Design of the Relativistic Heavy Ion Collider, BNL-52195 (Brookhaven National Laboratory, Upton, New York, 1989).

2. J.G. Cottiugham, AD/RHIC-39, 1988.

3. J.G. Cottingham, AD/RHIC/RD-19, 1990.

4. J.G. Cottingham, private communications, 1990.

5. J. Wei, Longitudinal Dynamics of the Non-Adiabatic Regime on Allcrnating-Gradient Synchrotrons, Ph.D thesis, State University of New York at Stony Brook, 1990. 